\title{
Determination of the Method of Construction of 1650 B.C. Wall Paintings
}

\author{
Constantin Papaodysseus, Dimitrios K. Fragoulis, Mihalis Panagopoulos, Thanasis Panagopoulos, \\ Panayiotis Rousopoulos, Mihalis Exarhos, and Angelos Skembris
}

\begin{abstract}
In this paper, a methodology of general applicability is presented for answering the question if an artist used a number of archetypes to draw a painting or if he drew it freehand. In fact, the contour line parts of the drawn objects that potentially correspond to archetypes are initially spotted. Subsequently, the exact form of these archetypes and their appearance throughout the painting is determined. The method has been applied to celebrated Thera Late Bronze Age wall paintings with full success. It has been demonstrated that the artist or group of artists has used seven geometrical archetypes and seven corresponding well-constructed stencils (four hyperbolae, two ellipses, and one Archimedes' spiral) to draw the wall painting "Gathering of Crocus" in 1650 B.C. This method of drawing seems to be unique in the history of arts and of great importance for archaeology, and the history of mathematics and sciences, as well.
\end{abstract}

Index Terms_-Image line pattern analysis, archaeological image edge analysis, archaeological object reconstruction, curve fitting, statistical pattern matching.

\section{INTRODUCTION}

$\mathrm{T}$ HE excavations at Akrotiri of the Greek island Thera (Santorini) in the Aegean Sea brought to light the ruins of a prehistoric city belonging to a civilization that flourished in the 17th century B.C., which corresponds to the Late Cycladic I period. Of all the finds unearthed at Akrotiri, the wall paintings constitute the most significant contribution to our knowledge of Aegean art, civilization, and society [2], [1].

According to prominent archaeologists, these wall paintings rank alongside the greatest archaeological discoveries. The late professor Marinatos originated the excavations in 1967, which are now being continued by Professor C. Doumas. The Akrotiri settlement ruins and, among them, the wall paintings have been very well-preserved since thick layers of volcanic ash covered the entire island, ensuring the abundant preservation of buildings and artifacts. The excavations at Akrotiri have so far brought to light about 10 houses or larger edifices, of which, only three have been fully explored. "Xeste 3" is the conventional name of a freestanding edifice, which lies in the southwestern part of the excavated settlement.

In the second floor of "Xeste 3," the east and northwest murals have been decorated with wall paintings whose iconographic program is comprised of horizontal bands in the upper and lower zone and, in the middle zone, the main theme, the celebrated

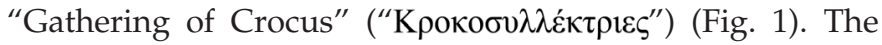
women engaged in this task wear a diversity of Minoan

- The authors are with the National Technical University of Athens, School of Electrical and Computer Engineering, 9 Heroon Polytechneiou, GR15773, Athens, Greece. E-mail: cpapaod@cs.ntua.gr, \{dfrag, saverios, panrous\}@mail.ntua.gr,\{mpanagop, mexarhos\}@central.ntua.gr,

thpanag@cs.ece.ntua.gr.

Manuscript received 10 Sept. 2004; revised 26 Aug. 2005; accepted 29 Dec. 2005; published online 13 July 2006.

Recommended for acceptance by D. Forsyth.

For information on obtaining reprints of this article, please send e-mail to: tpami@computer.org, and reference IEEECS Log Number TPAMI-0477-0904. costumes and are bedecked with precious ornaments (earrings, necklaces, and bracelets).

Archaeologists have noticed repetitions in the border lines of various wall paintings [3], but the deeper reason for these repetitions was completely unknown, as well as the actual method with which these wall paintings were drawn. Various disputes have emerged concerning the question if the paintings were drawn freehand or if guides (e.g., stencils) were employed by the artist(s). In this paper, a general methodology is introduced that has allowed for the determination of the method the artists(s) used to draw the wall painting "Gathering of Crocus." The method of construction is unique in the history of arts. Moreover, the results of this paper indicate that these wall paintings manifest a high drawing skill, as well as application and advanced sense of geometry and technology in this Late Bronze Age civilization.

\section{A First Stage Processing of the Wall PAINTING IMAGeS}

High-quality images of the wall painting in hand were obtained, along with a color palette and a scale so that proper processing could be applied later to compensate possible color and size discrepancies due to shooting conditions. The resolution of all obtained images is approximately 60 pixels per centimeter. Subsequently, we applied various image segmentation methods [10], [11], [12] to all preselected wall painting parts, each one corresponding to a different thematic unit. Thus, quite clear-cut and accurate region border extraction has taken place.

\subsection{Defining the Notion of a Wall Painting Object}

We have observed that each thematic unit consists of various drawn elements. For example, the female motif depicted in Fig. 1a consists of numerous elements such as:

1. the upper drawn line of the right forearm,

2. the stripes of the right lapel, 


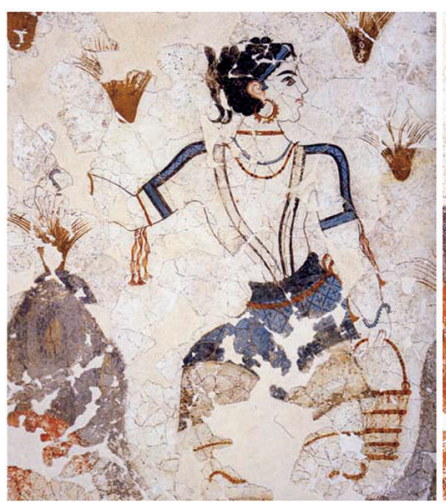

(a)

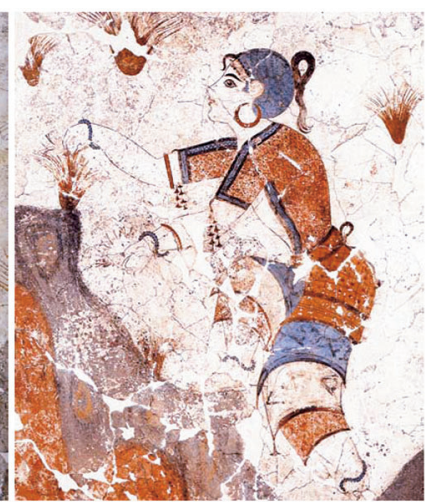

(b)
Fig. 1. Two female figures from the wall painting "Gathering of Crocus." Actual dimensions: (a) Height: $92.1 \mathrm{~cm}$, width: $73.5 \mathrm{~cm}$. (b) Height: $89.4 \mathrm{~cm}$, width: $74.4 \mathrm{~cm}$.

3. the left line of the left forearm,

4. the continuous drawn line consisting of the left side of the neck together with the left shoulder and the left line of the left arm,

5. the upper line of the waist,

6. the outer line of each hair lock, and

7. the continuous line consisting of the forehead and the upper line of the nose, etc.

The edge lines of each element are considered to be objects. For example, Element 2 of the aforementioned list corresponds to two objects, each being a continuous curve, as shown in Fig. 2a.

Thus, one can define the object to be a subset of the wall painting border, which represents a thematic unit, is smooth, and has as its beginning and end points discontinuities of the border line or of its first derivative. Careful examination of various objects indicates that they probably are a union of consecutive smooth curves to which the strict notion of object part corresponds, as will be defined in the following.

\subsection{Object Part Definition}

Suppose that the wall painting has suffered no wear and, therefore, that all objects' contour lines are continuous, described by the piecewise twice differentiable monoparametric vector equation $r(t)=x(t) \cdot \vec{i}+y(t) \cdot \vec{j}$. Then, an object part is defined to be a subset of the object curve whose beginning and end points are one of the following: 1) The beginning or the end of the object 2. 2) A point where the second derivative $r^{\prime \prime}(t)$ is discontinuous. 3) A point where the value of the determinant

$$
C=\left|\begin{array}{ll}
x^{\prime}(t) & y^{\prime}(t) \\
x^{\prime \prime}(t) & y^{\prime \prime}(t)
\end{array}\right|
$$

changes sign. The notion of an object part is crucial since, if the artist(s) indeed used stencils, then, each time he placed the stencil on the wall and drew a line, we consider he created an object part.

\subsection{Determination of the Object Parts in All Thematic Units of the Wall Painting}

In this section, mathematical methods are introduced for the determination of the endpoints of each object part. This

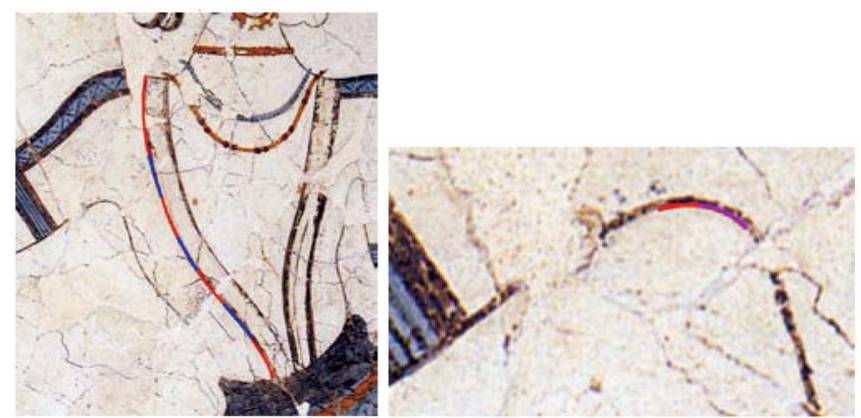

(a)

(b)

Fig. 2. Two elements corresponding to two objects and demonstration of the approximation with polynomials in order to determine the object parts. (a) Approximation of one object by a set of overlapping splines to determine points where a change of concavity occurs. (b) Local approximation of a subset of an object with third and fifth degree polynomials in order to determine points where a probable change of stencil occurs, without change of concavity.

is a crucial problem since, if the artist(s) used stencils to draw the wall paintings, then he/they, each time, placed the stencil on the wall and drew a concrete smooth line following the stencil, generating in this way well-defined object parts. Hence, if one wants to demonstrate that this was indeed the case, then one must determine a limited number of specific stencils that match the entire ensemble of object parts well.

If $L_{o}$ is the length of an arbitrary object, then we select an appropriate small percentage of $L_{o}$, say $L_{s}$. Subsequently, in order to spot points where there is a change of stencil together with a change of concavity, we proceed as follows: We divide all objects into consecutive pixel subsets of length $L_{s}$, slightly overlapping in $L_{O V}$ pixels, and we calculate the spline that best fits each such subset. In this way, one obtains an ensemble of consecutive, slightly overlapping splines $S_{i}, i=1,2, \ldots, N_{s}$, covering each object (see Fig. 2a). Notice that all small gaps of the various objects due to wear are bridged with this procedure. Good choices for $L_{s}$ and $L_{O V}$ are:

$$
\begin{gathered}
L_{s}=\left\{\begin{array}{l}
{\left[0.15 \cdot L_{o}\right], L_{o} \geq 400 \text { pixels }} \\
{\left[0.33 \cdot L_{o}\right], L_{o}<400 \text { pixels }}
\end{array}\right. \\
L_{o v}=\left\{\begin{array}{l}
{\left[0.09 \cdot L_{s}\right], L_{o} \geq 400 \text { pixels }} \\
{\left[0.18 \cdot L_{s}\right], L_{o}<400 \text { pixels },}
\end{array}\right.
\end{gathered}
$$

where, for any $x \in R,[x]$ stands for the integral part of $x$. Neighboring values of $L_{s}$ and $L_{O V}$ offer quite similar and satisfactory results.

Next, one computes the possible turning points of the splines $S_{i}$ and considers them as separation points of object parts. The turning points are determined via the standard method described in the object part definition Case 3 in Section 2.2.

To examine the case where a change of stencil occurs without change of concavity, we have proceeded as follows: For each object part of length $L$, we consider all its pixels naturally enumerated. For each such pixel $i$, where $L_{s} \leq i \leq L-L_{s}+1$, we apply the following method (see Fig. 2b): 
- We consider the pixels of ordinal number from $\left(i-L_{s}+1\right)$ up to $i$ and we compute the third degree polynomial (spline) $S_{i}$ best fitting them.

- We consider the pixels of ordinal number from $i$ up to $\left(i+L_{s}-1\right)$ and we compute the fifth degree polynomial $F_{i}$ best fitting them, where $F_{i}$ has the same value and the same first derivative with $S_{i}$ at the center of pixel $i$.

When pixel $i$ belongs to a specific object part, then, near pixel $i$, both at its left and its right, one expects that the object line will be described with the same functional form. Therefore, near pixel $i$, polynomials $S_{i}$ and $F_{i}$ that best fit this object line must also have the second order derivatives similar. On the other hand, when pixel $i$ is a point where a change of stencil occurs, one expects that, on the left and the right of this point, the object line will be described with a different function. Hence, one expects a relatively large discontinuity in the value of the second derivative. Thus, applying the aforementioned procedure to all corresponding pixels of an object, one may determine the points where a change of stencil occurs by demanding that the second derivative of $S_{i}$ and $F_{i}$ near $i$ differ more than a threshold $T_{S}$. It seems that a good estimate of $T_{S}$ can be obtained as follows: Let $\Delta_{i}^{(2)}$ be the difference of the second derivative of $S_{i}$ and $F_{i}$ at pixel $i$ of an object and let $\mu^{(2)}$ and $\sigma^{(2)}$ be the mean value and standard deviation of these $\Delta_{i}^{(2)}$. Since the pixels where a change of stencil occurs are far fewer in number than the pixels belonging to object parts, the value of $\Delta_{j}^{(2)}$ at a point $j$ where change of stencil occurs is expected to be "many $\sigma^{(2)}$ away from $\mu^{(2)}$." The performed experiments indicate that the choice of $T_{s}=\mu^{(2)}+3 \cdot \sigma^{(2)}$ offers a satisfactory determination of points where a change of stencil occurs. Rarely, one obtains a few more than one pixels, in which case, all these points are considered to be potential object part end points. The final decision about the exact point where a change of object part occurs is made by application of the method introduced in Section 4.3.

We would like to emphasize that, although a very good polynomial approximation of all objects can be achieved by the aforementioned technique, it is clear that these polynomials do not constitute stencils since their parameter values greatly vary from an object part to another.

\section{Defining a Class of Plausible Stencils}

The idea emerged among the authors that the artist(s) used prefabricated geometric stencils to draw the wall paintings. A first step to verify this conjecture is the determination of a set of geometric shapes whose conception and construction are not a priori prohibitive for the era, from an archaeological and historical point of view. For example, the linear spiral and the hyperbola can be constructed with the use of simple tools, even if this might require a considerable amount of novelty for the era.

Thus, extensive archaeological and historical analysis led to the conclusion that a set of geometric figures that could have been conceived and constructed by this civilization, are the following:

1. exponential spiral,

2. the spiral generated by unwrapping a thread around a peg, usually called the involute of a circle,

3. the linear or Archimedes' spiral,

4. the ellipse,
5. the parabola, and

6. the hyperbola.

A brief review of the mathematical history associated with these geometric curves is given below: According to various researchers [4], [6], [7], [9], [5], starting from Proclus

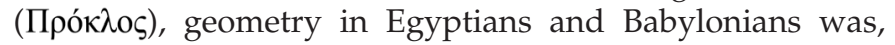
even in Thales' time, confined to an area computing stage

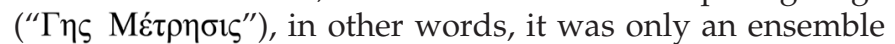
of empirical rules for computing surfaces, frequently with errors.

Concerning spirals, it is well-known that spiral shapes appear in various prehistoric civilizations even centuries before the prehistoric Thera civilization. There are infinitely many types of spirals. Among them, the involute of a circle and the exponential spiral are encountered in nature: The involute of a circle can be easily generated in everyday life events, while the exponential spiral can be found in various cockleshells. Thus, it is not a surprising fact that rough approximations of these two types of spirals are encountered quite early in various prehistoric civilizations.

On the other hand, the linear spiral seemingly does not exist in nature. In classical age geometry, the concept of the linear spiral is so far attributed to Konon (Kóvwv) from Samos in the third century B.C. Next, in "On Spirals," Archimedes defines the linear spiral and gives many fundamental properties and related theorems. For these reasons, the linear spiral also bears Archimedes' name [7], [9], [8], [6].

Concerning conics, it seems that the first who conceived them and realized that they result from the intersection of a cone with a plane was Menaichmos, around 350 B.C. The first who wrote about conics is Euclid around 300 B.C. According to Pappus (A.D. 320), "the four books of Euclid's Conics, were completed by Apollonius, who added four more books of Conics" [7], [9], [8], [6]. The names of the three conics' types (ellipse, hyperbola, and parabola), as well as many complicated theorems, are attributed to Apollonius.

\subsection{General Spiral Equations}

The general spiral equation is:

$$
\begin{gathered}
x(\theta)=x_{0}+R(\theta) * \cos \left(\alpha(\theta)-\phi_{0}\right), \\
y(\theta)=y_{0}+R(\theta) * \sin \left(\alpha(\theta)-\phi_{0}\right),
\end{gathered}
$$

where $x_{0}, y_{0}$ are the coordinates of the spiral center, $R(\theta)$ is any increasing function of $\theta$, and $\alpha(\theta)$ is any function of $\theta$, while $\phi_{0}$ accounts for a probable rotation of the spiral. The most celebrated spirals are the following, where their equations are written so as to incorporate the case where one deals with a part of the corresponding spiral that does not necessarily start at $\theta=0$ :

1. Archimedes' spiral, namely, the one with

$$
R(\theta)=k \cdot\left(\theta-\theta_{0}\right) \text { and } \alpha(\theta)=\left(\theta-\theta_{0}\right),
$$

where $k$ is a constant.

2. The involute of a circle with radius $r_{0}$, namely, the spiral generated when a thread wrapped around a peg is unwrapped. This spiral seems to be the simplest one to draw.

$$
\begin{aligned}
& R(\theta)=r_{0} \sqrt{1+\left(\theta-\theta_{0}\right)^{2}}, \\
& \alpha(\theta)=\left(\theta-\theta_{0}\right)-\arctan \left(\theta-\theta_{0}\right) .
\end{aligned}
$$


3. The exponential spiral satisfying: $R(\theta)=a \cdot e^{\beta \cdot\left(\theta-\theta_{0}\right)}$, $\alpha(\theta)=\left(\theta-\theta_{0}\right)$, where $\alpha, \beta$ are constants.

\subsection{Ellipse and Hyperbola: Useful, Relevant Characteristics}

The general equation of a conic in Cartesian coordinates is:

$$
\begin{aligned}
& A x^{2}+B y^{2}+C x y+D x+E y+F=0, \\
& A, B, C, D, E, F \in \mathbf{R}, \text { and } A^{2}+B^{2}+C^{2}>0 .
\end{aligned}
$$

One usually defines the discriminant $\Delta=4 \cdot A \cdot B-C^{2}$ and, as a consequence, if $\Delta>0$, the conic is an ellipse, if $\Delta<0$, the conic is a hyperbola, and, if $\Delta=0$, the conic is a parabola. If one performs an appropriate rotation of the coordinate axes of $\theta$ radians moving to a new pair of coordinates $(X, Y)$, then one can zero the coefficient $C$ of the cross term, in which case, one obtains the well-known normal equations of the three types of conics whose parameters are directly associated with their geometric characteristics.

\section{Testing if an Object Part Corresponds to a STENCIL}

\subsection{Choosing the Proper Stencil for Each Object Part and an Initial Estimation of Its Parameters}

Consider a drawn object part whose digitized image consists of $N^{P}$ pixels described by the sequence of vectors

$$
\overrightarrow{r_{i}^{P}}, i=1,2, \ldots, N^{P}
$$

starting at a reference center and pointing to each pixel center, where superscript $P$ stands for part. Suppose that one wants to test if this object part is the successful result of an artist's attempt to draw a geometrical prototype described by the parametric vector equation

$$
\overrightarrow{r^{M}}(\theta \mid \Pi),
$$

where $\theta$ is the independent variable, $\Pi$ is the curve set of parameters, and superscript $M$ stands for model. For example, for the linear spiral polar parametric equation,

$$
\begin{aligned}
\overrightarrow{r^{M}}(\theta \mid \Pi)= & \left(x_{0}+k \cdot\left(\theta-\theta_{0}\right) \cdot \cos \left(\theta-\theta_{0}+\varphi_{0}\right)\right) \vec{i} \\
& +\left(y_{0}+k \cdot\left(\theta-\theta_{0}\right) \cdot \sin \left(\theta-\theta_{0}+\phi_{0}\right)\right) \vec{j},
\end{aligned}
$$

$\theta \in \mathbf{R}$ is the independent variable (the polar angle) and $\Pi=$ $\left\{x_{0}, y_{0}, k, \phi_{0}, \theta_{0}\right\}$ is the spiral set of parameters.

Next, we compute the optimal set of parameters $\Pi^{\circ}$ and the corresponding sequence of values of the independent variable $\theta_{i}, i=1,2, \ldots, N^{P}$, so that

$$
\overrightarrow{r^{M}}\left(\theta_{i} \mid \Pi^{o}\right)
$$

best fits $\overrightarrow{r_{i}^{P}}$ according to a chosen norm L. Algorithms to achieve this are the well-known conjugate gradient or the easier to implement Nelder-Mead method, starting from a tentative set of values of $\Pi$ and letting $\Pi$ converge to $\Pi^{\circ}$ so that $\mathrm{L}$ is minimized. For example, for a given object part$$
\overrightarrow{r_{i}^{P}}
$$

and a specific potential stencil described by $\overrightarrow{r^{M}}$, we minimize the quantity

$$
E_{2}=\sum_{i=1}^{N^{P}}\left\|r_{i}^{P}-r_{i}^{M}\right\|=\sum_{i}^{N^{P}}\left(\left(x_{i}^{P}-x_{i}^{M}\right)^{2}+\left(y_{i}^{P}-y_{i}^{M}\right)^{2}\right) .
$$

For all obtained object parts of each wall painting, we have applied the aforementioned procedure, considering, each time $\overrightarrow{r^{M}}$ to be: Archimedes' spiral, the involute of a circle, the exponential spiral, and all three conics. In this way, for each object part of the drawing and for each potential stencil, we have obtained a set of corresponding initial parameters that offer a first, not yet optimal, minimum for the error. The nonoptimality is due to the intrinsic ineffectiveness of the minimization procedure. The relatively large number of curve parameters, as well as the considerably large number of pixels $N^{P}$ of the object part introduce a noticeable complexity in the minimization procedure, which makes the employed algorithm converge to a local minimum near the absolute minimum but different from that. This occurs almost always in practice, independently of the type of the employed minimization algorithm. Thus, for each object part of the wall painting in hand one can determine the potential stencil corresponding to the minimum error among the six error values computed with the aforementioned procedure. This minimum error prototype constitutes a first candidate stencil, which the artist probably used to draw the specific object part. The corresponding optimal set of parameters is a first estimation of the characteristics of this candidate stencil.

Notice that the Hough transform also offered quite rough results in the attempt to determine the stencils and obtain a first estimation of their parameters [18]. In general, the problem faced in this paper is not a typical curve-fitting problem like the ones tackled in [13], [14], [15], [16], [17]. On the contrary, one can define the problem in hand as follows: Try to figure out probable prototypes used by the artist(s) to draw a specific painting, specify their primary parameters, and establish the corresponding stencils used for drawing the painting, with the maximum possible accuracy.

\subsection{Stencil Confirmation and Optimization of Its Parameters for Each Object Part}

In this section, we will introduce a method in order to irrevocably decide if the previously estimated minimum error prototype indeed corresponds to the object part in hand and, in addition, to obtain a more accurate estimation of its parameters.

Thus, consider a potential stencil $\overrightarrow{r^{M}}\left(\theta_{i} \mid \Pi^{o}\right)$, where $\Pi^{o}$ is the initial estimation of its parameters, as computed in Section 4.1. We stress that, among these parameters, there is a subset that determines the prototype form, while the remaining parameters determine its position in the plane. For example, $k$ determines the form of the linear spiral, $r_{o}$ the exact shape of the involute of a circle, $\alpha, \beta$ the form of the exponential spiral, $a, b$ the shape of ellipse or hyperbola, and $a, b, c$ the parabola form. We will refer to these parameters with the name "primary parameters" of the prototype.

Next, for each of the six potential stencils with optimal primary parameters $\Pi_{p}^{o}$, one defines a subdomain around the 
$\Pi_{p}^{o}$ values in order to achieve the optimal estimation of these values in this subdomain. For clarity, we will construct this subdomain in a specific example case first: In fact, suppose that the potential stencil in hand is a hyperbola with initially estimated optimal primary parameters $a^{o}, b^{o}$. Next, we define two intervals around them: $\left[a^{o} \cdot\left(1-p_{a}\right), a^{o} \cdot\left(1+p_{a}\right)\right]$ and $\left[b^{o} \cdot\left(1-p_{b}\right), b^{o} \cdot\left(1+p_{b}\right)\right]$, where $p_{a}, p_{b}$ are properly chosen factors. All performed experiments indicate that a very satisfactory choice for the present application is $p_{a}=$ $p_{b}=0.25$. Moreover, one defines a partition of these intervals to obtain two sequences $I^{a}, I^{b}$ of $K_{a}$ and $K_{b}$ points, respectively, such that $a^{o} \in I^{a}$ and $b^{o} \in I^{b}$. Subsequently, via the Cartesian product $I^{a} \times I^{b}$ of these sequences, we obtain $K_{a} \cdot K_{b}$ values of the corresponding hyperbola primary parameters.

Each pair $\left(I_{\lambda}^{a}, I_{\mu}^{b}\right)$, corresponds to a specific prototype hyperbola to which the object part in hand must fit optimally. To achieve this optimal fitting, the following lemmas will prove useful:

Lemma 1. Consider two discrete $N$-point curves in the same plane, say $\left(x_{i}, y_{i}\right)$ and $\left(P_{i}, Q_{i}\right)$, with arbitrary orientation. Suppose that one wants to estimate the optimum rotation angle $\theta$ and optimal translation $\left(x_{0}, y_{0}\right)$ so as to fit curve $\left(x_{i}, y_{i}\right)$ to curve $\left(P_{i}, Q_{i}\right)$ in the Least Squares sense. Equivalently, if $\left(X_{i}, Y_{i}\right)$ is the result of the rotation and translation of curve $\left(x_{i}, y_{i}\right)$, we demand quantity $E_{2}^{\prime}=\sum_{\text {alli }}\left\{\left(X_{i}-P_{i}\right)^{2}+\left(Y_{i}-Q_{i}\right)^{2}\right\}$ to be minimum. After setting the corresponding partial derivatives to zero and some calculus, one obtains:

$$
\begin{aligned}
x_{0}= & \frac{1}{N} * \sum_{i=1}^{N}\left(P_{i}-\delta x_{i}\right), y_{0}=\frac{1}{N} * \sum_{i=1}^{N}\left(Q_{i}-\delta y_{i}\right), \\
\tan (\theta)= & \frac{\sum_{i=1}^{N}\left(x_{i} \cdot \delta Q_{i}-y_{i} \cdot \delta P_{i}\right)}{\sum_{i=1}^{N}\left(x_{i} \cdot \delta P_{i}+y_{i} \cdot \delta Q_{i}\right)}
\end{aligned}
$$

where $\delta x_{i}=-y_{i} \cdot \sin (\theta)+x_{i} \cdot \cos (\theta), \delta y_{i}=y_{i} \cdot \cos (\theta)+x_{i}$. $\sin (\theta), \delta P_{i}=\frac{1}{N} \sum_{j=1}^{N} P_{j}-P_{i}$, and $\delta Q_{i}=\frac{1}{N} \sum_{j=1}^{N} Q_{j}-Q_{i}$.

Lemma 2. Now, in order to fit a given object part of $L$ pixels $\left(x_{i}, y_{i}\right)$ to the best position of a model potential stencil, one proceeds as follows:

1. Frequently, the L pixels of the object part as obtained by the segmentation method are very dense and misplaced so that it is very difficult to achieve one-to-one correspondence between these pixels and points of the potential stencil. To circumvent this difficulty, we choose $N$ pixels of the object part so that two successive pixels are separated by a small number of object part pixels, say three or four. In the following, when we refer to the $N$-pixel object part, we mean the aforementioned subset of the object part pixels. Subsequently, one computes the Euclidean distance of all pairs of successive object part pixels; we let $d_{j}^{P}$ be the Euclidean distance between the $j$ th and the $j+1$ th pixels of the object part and $D(\mathrm{M}, \Lambda)$ be the Euclidean distance between any two points $\mathrm{M}$ and $\Lambda$.

2. Consider an arbitrary potential stencil with independent variable $t$. Then, one creates a set of points belonging to the potential stencil, starting at $t_{0}$ and ending at $t_{e}$, being as dense as possible. In other words, one first generates a sequence of points of the potential stencil $P^{s}\left(t_{i}\right), t_{i} \in\left[t_{0}, t_{e}\right]$, such that two successive points have a very small distance, much smaller than the pixel dimensions.

3. For each point $n$ of the sequence $P^{s}\left(t_{i}\right)$, one creates an $\mathrm{N}$-vertices polygonal line (poly-line) starting at the point $P^{s}\left(t_{n}\right)$ in hand, which we call $M_{1}^{n}$, where its subsequent vertices are defined as follows:

The second vertex $M_{2}^{n}$ is the point $P^{s}\left(t_{k}\right)$, for which the following relations hold:

$$
\begin{aligned}
& D\left(P^{s}\left(t_{k}\right), M_{1}^{n}\right) \geq d_{1}^{P}, D\left(P^{s}\left(t_{k-1}\right), M_{1}^{n}\right)<d_{1}^{P}, \\
& D\left(P^{s}\left(t_{k}\right), M_{1}^{n}\right)-d_{1}^{P} \leq d_{1}^{P}-D\left(P^{s}\left(t_{k-1}\right), M_{1}^{n}\right) .
\end{aligned}
$$

Clearly, if inequalities (2) hold, while $D\left(P^{s}\left(t_{k}\right), M_{1}^{n}\right)-$ $d_{1}^{P}>d_{1}^{P}-D\left(P^{s}\left(t_{k-1}\right), M_{1}^{n}\right)$, then $M_{2}^{n}=P^{s}\left(t_{k-1}\right)$.

Next, the $M_{j}^{n}$ vertex is inductively defined via the analogous relations:

$$
\begin{aligned}
& D\left(P^{s}\left(t_{k}\right), M_{j-1}^{n}\right) \geq d_{j-1}^{P}, D\left(P^{s}\left(t_{k-1}\right), M_{j-1}^{n}\right)<d_{j-1}^{P} . \\
& \quad \text { If }
\end{aligned}
$$$$
D\left(P^{s}\left(t_{k}\right), M_{j-1}^{n}\right)-d_{j-1}^{P} \leq d_{j-1}^{P}-D\left(P^{s}\left(t_{k-1}\right), M_{j-1}^{n}\right),
$$

then $M_{j}^{n}$ is $P^{s}\left(t_{k}\right)$, else if

$$
D\left(P^{s}\left(t_{k}\right), M_{j-1}^{n}\right)-d_{j-1}^{P}>d_{j-1}^{P}-D\left(P^{s}\left(t_{k-1}\right), M_{j-1}^{n}\right),
$$

then $M_{j}^{n}$ is $P^{s}\left(t_{k-1}\right)$.

4. For all $n$, we apply Lemma 1 , where $\left(x_{i}, y_{i}\right)$ are the coordinates of the center of the $N$ pixels belonging to the object part in hand, while $\left(P_{i}, Q_{i}\right)$ are the coordinates of the model vertices $M_{1}^{n}, M_{2}^{n}, \ldots, M_{N}^{n}$. In this way, for each point $P^{s}\left(t_{n}\right)$ of the specific potential stencil, one obtains an error $E_{2}^{n}$ describing the way the object part best fits to the specific model poly line. Clearly, the minimization of $E_{2}^{n}$, for all points $P^{s}\left(t_{n}\right)$ for which the model poly line can be constructed, offers the position, i.e., the value of $n$ in which the object part in hand best fits the specific potential stencil. The corresponding minimum value of $E_{2}^{n}$ is a measure of the goodness of fit of these two sets of points.

At this point, we can unambiguously compute the angle of rotation and parallel translation that one must apply to the sequence of points $\left(x_{i}, y_{i}\right)$ belonging to the specific object part so that it best fits the potential stencil with primary parameters $\left(I_{\lambda}^{a}, I_{\mu}^{b}\right)$ by direct application of Lemmas 1 and 2 . This procedure will also furnish the corresponding minimum average error $\varepsilon_{\lambda, \mu}$ as well as the exact fitting position.

The minimum $\varepsilon_{\lambda, \mu}$ value for all pairs of primary parameters $\left(I_{\lambda}^{a}, I_{\mu}^{b}\right)$ offers a better and far more reliable estimation of the optimal primary parameters of the stencil in hand.

Recursive application of this procedure using subdomains of continually smaller size and continually better refinement of the corresponding intervals $I^{a}, I^{b}$ offers convergence to optimal primary parameters of the considered potential stencil.

An analogous procedure to the aforementioned one is applied to the other five potential stencils, too, in order to 


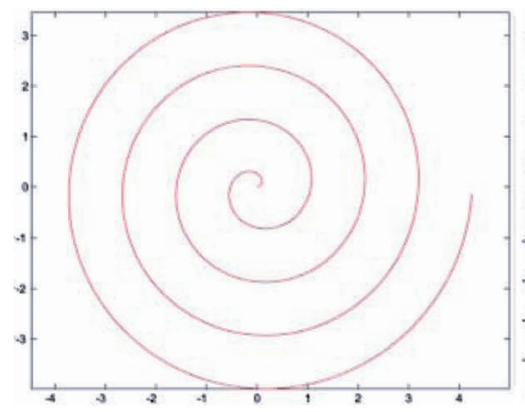

(a)

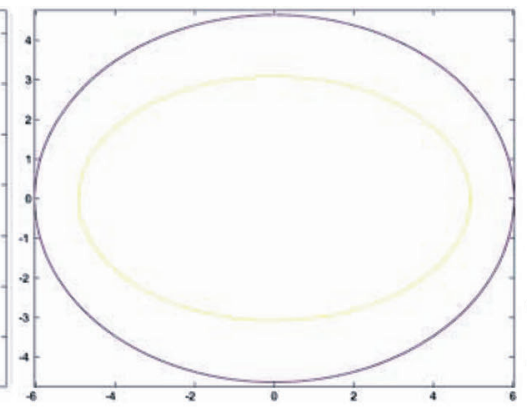

(b)

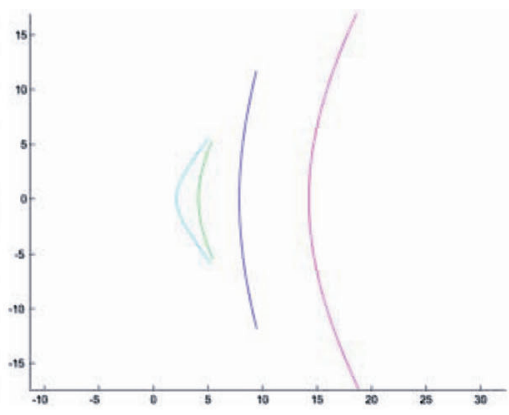

(c)

Fig. 3. The seven stencils that presumably have made the wall painting "Gathering of Crocus" shown in Fig. 1 and Fig. 2. All axes are scaled in centimeters. (a) The linear (Archimides') spiral, always in red. (b) Ellipse 1 in yellow and ellipse 2 in purple. (c) Hyperbola 1 always in magenta, hyperbola 2 always plotted in green, hyperbola 3 in blue, and hyperbola 4 always in cyan.

associate with each object part and each potential stencil the best fitting parameters and the corresponding minimum error, which is an actual measure of the way the specific potential stencil best fits the object part in hand. In this way, one associates six fitting error values with each object part and six corresponding sets of best fitting parameters.

For all object parts, there is a clear-cut minimum among the six best fitting error values; we consider that the prototype corresponding to this minimum error is the type of stencil the artist(s) employed to draw the corresponding object part c.1650 B.C.

We stress that, for all object parts of the celebrated wall painting of "Gathering of Crocus," the minimum error occurred only for three types of stencils: hyperbolas, ellipses, and linear spirals. This means that the artist(s) used stencils corresponding only to these three types of curves.

\subsection{Determining the Set of Stencils Used for Drawing the Wall Paintings}

First, we classify all object parts according to if they best fit to a hyperbola or to an ellipse or to a linear spiral. One expects that the artist(s) used a limited number of stencils to draw the wall paintings. On the other hand, let as suppose that two object parts were drawn using the same stencil, say a hyperbola, with primary parameters $a_{1}, b_{1}$. If one applies the methodology introduced in Sections 4.1 and 4.2 to these two object parts, then one will obtain two different pairs of primary parameters of the best fitting hyperbola close to $a_{1}, b_{1}$ but different from them, in general. This discrepancy is most probably due to slight random movements of the paintbrush, the variable line width, the wear the wall paintings have suffered, the finite images resolution, etc.

Therefore, special effort should be made in order to determine the number of different stencils the artist used, as well as a consistent estimation of the primary parameters of these stencils. In order to achieve that, we proceed as follows:

Consider first all object parts corresponding to the hyperbola stencil, as deduced in Section 4.2. In the $(a, b)$ plane, one places all primary parameters of the best fitting hyperbola to each object part separately. In this way, one obtains four disjoint sets of points, where each set consists of adjacent points. It is logical to assume that each such set corresponds to a different hyperbola stencil. Let $N 1$, $N 2, N 3$, and $N 4$ be the number of points of each set; we stress once more that each such point corresponds to a specific object part and to the pair of the hyperbola primary parameters best matching to it. In order to estimate the parameters of each one of the four stencils, we proceed as in Section 4.2.

For each one of the four sets, we define a rectangular region containing all points of the set and then we define a dense partition $I^{a} \times I^{b}$ of this region. For each partition point $\left(I_{\lambda}^{a}, I_{\mu}^{b}\right)$, one computes the aggregate error $\gamma_{\lambda, \mu}^{a g g}=\sum_{j=1}^{N 1} \varepsilon_{\lambda, \mu}^{j}$, where $\varepsilon_{\lambda, \mu}^{j}$ is the average error of approximation of the $j$ th object part by the hyperbola with primary parameters $\left(I_{\lambda}^{a}, I_{\mu}^{b}\right)$. The minimum of these aggregate errors for all partition points $\left(I_{\lambda}^{a}, I_{\mu}^{b}\right)$ is considered to define the final primary parameters of the first hyperbola stencil. Similarly, one can calculate the final primary parameters of the other three hyperbola stencils.

Proceeding along similar lines, one can demonstrate that there are two different elliptical stencils and one can estimate their final primary parameters.

The linear spiral case is similar and actually simpler since there is only one primary parameter, $k$. The related analysis shows that there is a large class of object parts corresponding to a single linear spiral stencil. All seven of these stencils are shown in Fig. 3, plotted in their characteristic color; as described in Table 1.

TABLE 1

The Primary Parameters of the Seven Stencils, Together with the Colors Associated with Each Stencil in Figs. 3, 4, 5, 6, 7, and 8

\begin{tabular}{|c|c|c|c|}
\hline Type of stencil & $\begin{array}{c}\text { Color in figures } \\
\mathbf{3} \mathbf{4} \mathbf{5}, \mathbf{6}\end{array}$ & \multicolumn{2}{|c|}{$\begin{array}{c}\text { Primary parameters } \\
\text { [cm] }\end{array}$} \\
\hline hyperbola 1 & magenta & $\mathrm{a}=14.24$ & $\mathrm{~b}=20.12$ \\
\hline hyperbola 2 & green & $\mathrm{a}=4.11$ & $\mathrm{~b}=6.29$ \\
\hline hyperbola 3 & blue & $\mathrm{a}=7.86$ & $\mathrm{~b}=17.63$ \\
\hline hyperbola 4 & cyan & $\mathrm{a}=2.09$ & $\mathrm{~b}=2.52$ \\
\hline ellipse 1 & yellow & $\mathrm{a}=4.64$ & $\mathrm{~b}=6.03$ \\
\hline ellipse 2 & purple & $\mathrm{a}=10.85$ & $\mathrm{~b}=7.96$ \\
\hline linear spiral & red & \multicolumn{2}{|c|}{$\mathrm{k}=0.169$} \\
\hline
\end{tabular}


TABLE 2

Indicative Values Manifesting the Way in Which the Stencils Match the Corresponding Objects Parts

\begin{tabular}{|c|c|c|c|c|}
\hline \begin{tabular}{|c|} 
NAME- \\
OBJECT \\
PART \\
DESCRIPTION
\end{tabular} & $\begin{array}{l}\text { OBJECT } \\
\text { PART } \\
\text { LENGTH } \\
(\mathrm{mm})\end{array}$ & $\begin{array}{c}\text { MEAN } \\
\text { ERROR } \\
\text { VALUE } \\
(\mathrm{mm}) \\
\end{array}$ & $\begin{array}{l}\text { STD OF } \\
\text { THE } \\
\text { ERROR } \\
(\mathrm{mm}) \\
\end{array}$ & \\
\hline \multicolumn{5}{|c|}{ Figure 5} \\
\hline $\begin{array}{c}\text { Upper sash } \\
\text { boundary }\end{array}$ & 151.9 & 0.41 & 0.53 & ellipsis 2 (purple) \\
\hline $\begin{array}{c}\text { Lady's left } \\
\text { shoulder upper } \\
\text { boundary }\end{array}$ & 136.1 & 0.29 & 0.43 & hyperbola 2 (green) \\
\hline $\begin{array}{l}\text { Lower sash } \\
\text { boundary }\end{array}$ & 134.2 & 0.42 & 0.63 & ellipsis 2 (purple) \\
\hline \begin{tabular}{|c|} 
Orange (lower) \\
necklace \\
boundary
\end{tabular} & 118.5 & 0.50 & 0.66 & ellipsis 1 (yellow) \\
\hline \begin{tabular}{|l|} 
Left shoulder \\
lower boundary
\end{tabular} & 115.6 & 0.39 & 0.58 & hyperbola 4 (cyan) \\
\hline \begin{tabular}{|c|} 
Left lapel outer \\
boundary part \\
1
\end{tabular} & 103.5 & 0.39 & 0.54 & $\begin{array}{c}\text { hyperbola } 1 \\
\text { (magenta) }\end{array}$ \\
\hline \begin{tabular}{|c|} 
Middle (blue) \\
necklace \\
boundary
\end{tabular} & 92.3 & 0.57 & 0.70 & ellipsis 1 (yellow) \\
\hline \begin{tabular}{|c|}
$\begin{array}{c}\text { Right forearm } \\
\text { upper } \\
\text { boundary }\end{array}$ \\
\end{tabular} & 82.6 & 0.28 & 0.45 & $\begin{array}{c}\text { hyperbola } 1 \\
\text { (magenta) }\end{array}$ \\
\hline $\begin{array}{l}\text { Left forearm } \\
\text { rightmost } \\
\text { boundary }\end{array}$ & 82.6 & 0.51 & 0.48 & $\begin{array}{c}\text { hyperbola } 1 \\
\text { (magenta) }\end{array}$ \\
\hline $\begin{array}{c}\text { Left lapel outer } \\
\text { boundary part } \\
1\end{array}$ & 77.0 & 0.43 & 0.56 & $\begin{array}{c}\text { hyperbola } 1 \\
\text { (magenta) }\end{array}$ \\
\hline \multicolumn{5}{|c|}{ Figure 6} \\
\hline Hunch & 220.3 & 0.52 & 0.19 & $\begin{array}{c}\text { hyperbola } 1 \\
\text { (magenta) }\end{array}$ \\
\hline \begin{tabular}{|l|} 
Third from top \\
trouser stripe \\
\end{tabular} & 136.9 & 0.42 & 0.55 & ellipsis 2 (purple) \\
\hline \begin{tabular}{|c|}
$\begin{array}{c}\text { Second from } \\
\text { top trouser } \\
\text { stripe }\end{array}$ \\
\end{tabular} & 136.6 & 0.47 & 0.74 & ellipsis 2 (purple) \\
\hline \begin{tabular}{|c|}
$\begin{array}{c}\text { Right forearm } \\
\text { upper } \\
\text { boundary }\end{array}$ \\
\end{tabular} & 125.3 & 0.29 & 0.56 & $\begin{array}{c}\text { hyperbola } 1 \\
\text { (magenta) }\end{array}$ \\
\hline Rear end & 103.8 & 0.68 & 0.76 & ellipsis 2 (purple) \\
\hline $\begin{array}{l}\text { First from top } \\
\text { trouser stripe }\end{array}$ & 102.4 & 0.52 & 0.68 & ellipsis 2 (purple) \\
\hline \begin{tabular}{|c|} 
Left forearm \\
lower boundary
\end{tabular} & 95.0 & 0.35 & 0.61 & $\begin{array}{c}\text { hyperbola } 1 \\
\text { (magenta) }\end{array}$ \\
\hline \begin{tabular}{|c|} 
Uppermost \\
head boundary \\
line
\end{tabular} & 94.9 & 0.25 & 0.39 & spiral (red) \\
\hline Front left hip & 89.4 & 0.60 & 0.76 & ellipsis 2 (purple) \\
\hline
\end{tabular}

The term "error" stands for the Euclidian distance of the proper contou part from the corresponding stencil; it is measured in millimeters. The values inside parentheses are the corresponding mean error and standard deviation when the wall paintings wear is neglected.

By applying the method introduced in Section 4.2, we have fit all lines of the women figures appearing in "Gathering of Crocus" to the obtained set of stencils, with an exceptionally low error. This error is estimated as the distance of the contour pixels centres of each object part from the matching geometric prototype curve. In fact, Table 2 shows the way a number of long object parts is approximated by the stencils; we stress that these errors are essentially the larger ones. The overall approximation error of all object parts is around $3.3 \cdot 10^{-4} \mathrm{~m}$ or, equivalently, of a millimeter per pixel, while the overall standard deviation is 0.54 .

In addition, we would like to point out that 76 object parts have been generated by the linear spiral prototype always plotted in red, 35 by hyperbola 1 shown in magenta, seven by hyperbola 2 always in green, 15 by hyperbola 3 always in cyan, 16 by hyperbola 4 always in blue, two by ellipse 1 always in yellow, and, finally, 11 by ellipse 2 always in purple. Overall, there are 90 stencil realizations in the female motif of Fig. 5 and 72 in the female motif of Fig. 6. It might be intuitively evident that the longer the stencil part corresponding to a specific object part, the greater the probability that the corresponding contour indeed matches to this prototype. However, a rigorous treatment of this statement is well outside the scope of the present paper and, consequently, it will be attempted in another one. In any case, employing the aforementioned results, one may confirm the statistical hypothesis that the object parts indeed correspond to the proper stencils; the details of this confirmation are not included here for reasons of brevity. Using the final primary parameters of each stencil, as well as a maximum allowed error of discrepancy between the object part and the corresponding stencil, we have confirmed or irrevocably defined the limits of each object part.

\subsection{The Most Probable Method of Drawing the Considered Wall Paintings}

The particularly low fitting error between stencils and the corresponding objects parts strongly supports the hypothesis that the artist(s) indeed used the estimated seven stencils to draw this wall painting. In other words, the artist(s) had these prefabricated geometric curves at his disposal and used one of them each time as a guide to draw the lines, which, in this paper, are described as object parts. With artfulness, the artist(s) succeeded in obtaining smooth line continuation at the points where change of the stencil or of its positioning on the wall occurred. We emphasize that the artist(s) did not use the entire stencil each time, but, instead, he/they used the part of the corresponding stencil he/they considered most suitable. Fig. 4 shows the ensemble of object parts matching to hyperbola 1, drawn in black, as well as their positioning on the prototype. In general, the average length of the employed seven stencil subsets is around $6 \mathrm{~cm}$.

One plausible explanation for the employment of this method is that, in this way, the artist(s) achieved a particularly steady line of the drawn objects and speed of execution, which is frequently crucial for the fresco technique.

The validity of the aforementioned statements is also demonstrated in Figs. 5 and 6 where each one of the seven stencils approximating the corresponding object part is shown by a different color. The remarkable precision with which the specific stencils fit the corresponding line contours is shown in Figs. 7 and 8, depicting details extracted from the wall paintings in hand.

Moreover, we would like to point out that the existence of four different hyperbola stencils, as well as of two different ellipse stencils indicates that these geometric curves have not been drawn and constructed nor accidentally either by hand. On the contrary, the appearance of the same functional forms with different parameters practically proves that a person or a group of people had a concrete method of constructing these 


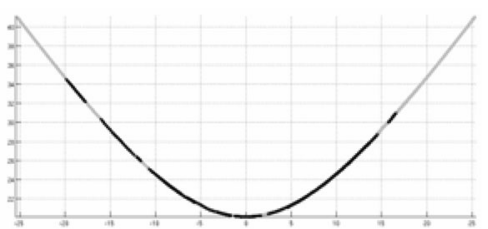

(a)

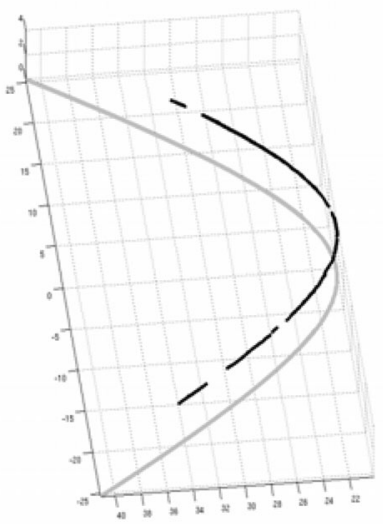

(b)

Fig. 4. Hyperbola 1 in black with a large number of object parts it optimally matches placed on it exactly. The object parts are shown in gray. Here, both a two-dimensional and a pseudo-three-dimensional representation are shown.

forms almost 1,300 years before their foundation is reported in the classical ages.

In addition, the fact that the various object parts approximate the seven aforementioned geometric curves (stencils) with impressive precision strongly indicates that there was a very neat and precise method of drawing these geometric figures and constructing the corresponding stencils. One cannot exclude that the method of drawing these curves was a geometric one.

Finally, the method of drawing the wall painting described in this paper seems to be unique in the history of arts. The construction of precise, advanced for the era, geometric stencils and their subsequent use in order to depict beautiful figures and motifs requires a remarkable sense of regularity, proportionality, symmetry, and geometry. The fact that this aptness emerged in the Late Bronze Age civilization of Thera c. 3,700 years ago makes the whole subject even more impressive.

\subsection{Confirmation that a Set of Wall Paintings Was Drawn by Hand and Not by Stencils}

In order to demonstrate the antithesis of paintings drawn by hand on one side and those drawn with the use of guides (stencils) on the other, we looked for other wall paintings that intuitively gave us the feeling they were handmade. These wall paintings, shown in Fig. 9 and called "Ikria," are of similar dimensions to the "Gathering of Crocus" and initially decorated the walls of rooms 4 and $4 \mathrm{~b}$ of the "West House."

A first step toward proving the conjecture that the "Ikria" are handmade is to exclude the case that there are noticeable and consistent repetitions of contour lines in them. In fact,

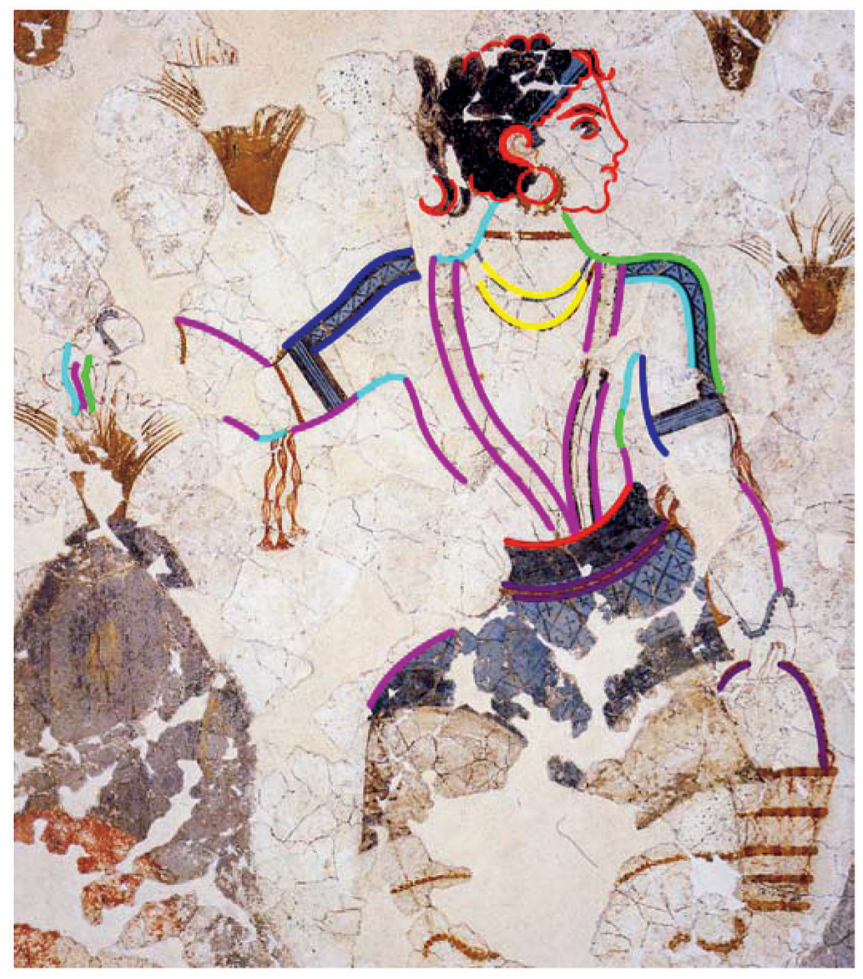

Fig. 5. Demonstration of the excellent way the geometric stencils described in the paper approximate the corresponding object parts of the female motif depicted in Fig. 1a. Each color corresponds to a different stencil, as indicated in Fig. 3 and Table 1. Concisely: 1) magenta, blue, green, and cyan are hyperbolas of different primary parameters each, 2) yellow is an ellipse of different primary parameters than the purple one, and 3 ) red is a single linear spiral. Notice that identical colors in Figs. 3, 4, 5, 6,7 , and 8 correspond to the same stencils.

consider the three entire "sinusoidal" arcs belonging to this wall painting, shown in Fig. 9. We considered each of these arcs as an independent model say, $A_{1}, A_{2}, A_{3}$. Next, we applied the Hough transform three times, once for each model, looking for patterns identical to it or to any of its subparts of length $4 \mathrm{~cm}$ or more. Hough's transform has shown the following:

1. The arcs were by no means repeated as a whole.

2. A very limited number of subparts of one arc, say $A_{1}$, have been spotted in the other arcs.

3. The length of these common subparts of $A_{1}, A_{2}, A_{3}$ never exceeded $5.5 \mathrm{~cm}$.

4. The common subparts could not cover the full length of the arcs nor even a noticeable portion of them.

We repeated the aforementioned procedure using as a model the mirrored versions of these arcs and all results were analogous.

On the contrary, when the aforementioned approach was applied to the wall paintings of Fig. 1, a large number of contour parts of length greater than $5 \mathrm{~cm}$ appearing in these figures was verified. Certain of these repetitions were of considerable length (e.g., see the table repetitions in Table 2): For example, consider the lady's hunch in Figs. $1 \mathrm{~b}$ and 6. Ignoring the exact form of the stencils for a moment, we have considered this object part, as it resulted from the segmentation process, to be a model discrete curve, say $H$. When the Hough transform was applied to the images of Fig. 1, using $H$ as a prototype, and once more looking for realizations of 


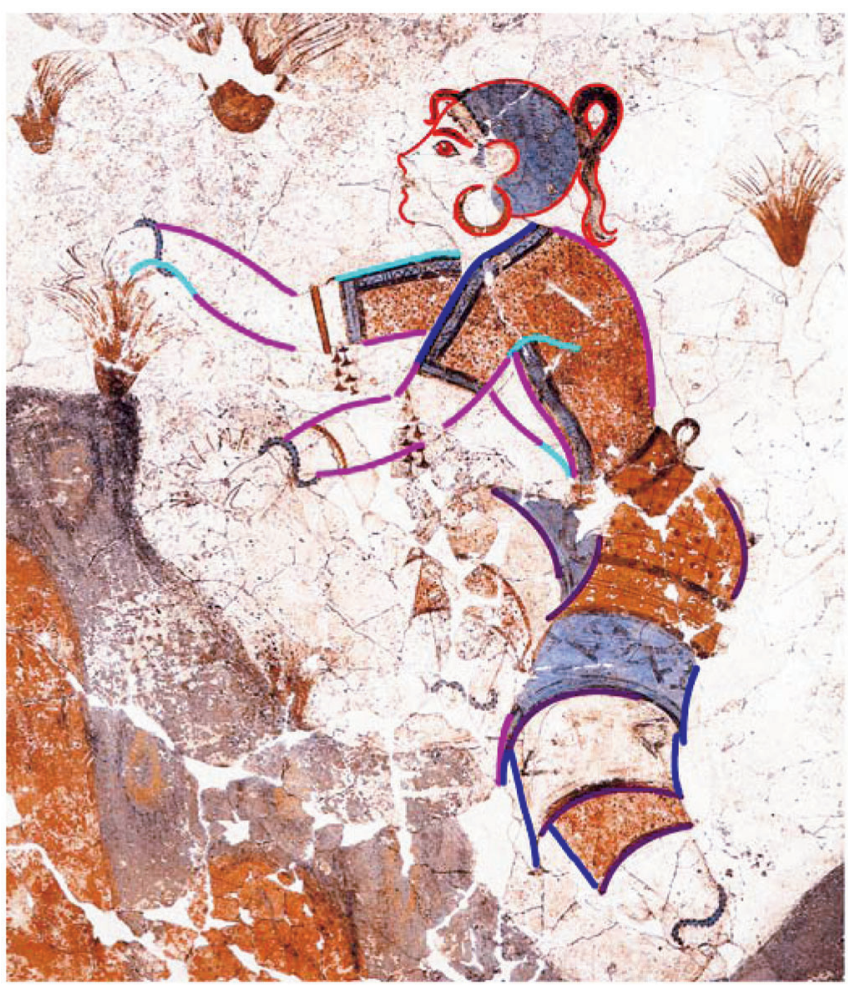

Fig. 6. Demonstration of the excellent way the geometric stencils described in the paper approximate the corresponding object parts of the female motif depicted in Fig. 1b. Each color corresponds to a different stencil as indicated in Fig. 3 and Table 1. Concisely, 1) magenta, blue, green, and cyan are hyperbolas of different primary parameters each, 2) purple is an ellipse of different primary parameters than the purple one, and 3 ) red is a single linear spiral. Notice that identical colors in Figs. 3, 4, 5, 6,7 , and 8 correspond to the same stencils.

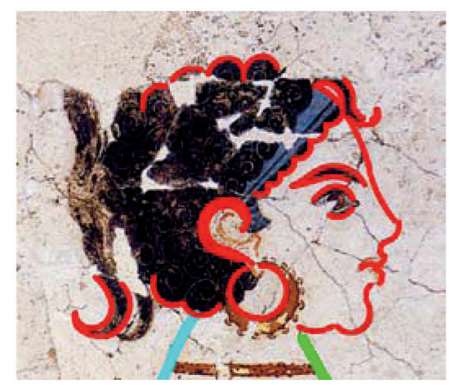

Fig. 7. Detail extracted from Fig. 5 in order to demonstrate the excellent way in which the geometric stencils correspond to the object parts. The same convention with Figs. 3, 4, 5, 6, 7, and 8 concerning colors holds.

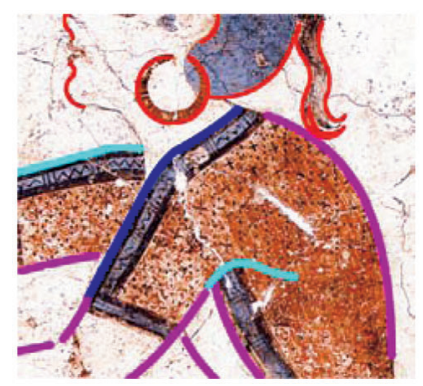

Fig. 8. Detail extracted from Fig. 6 in order to demonstrate the excellent way in which the geometric stencils correspond to the object parts. The same convention with Figs. 3, 4, 5, 6, 7, and 8 concerning colors holds.

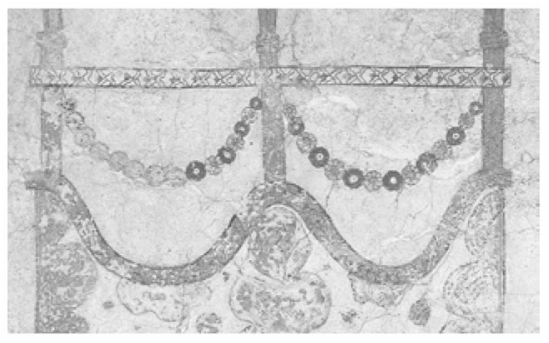

(a)
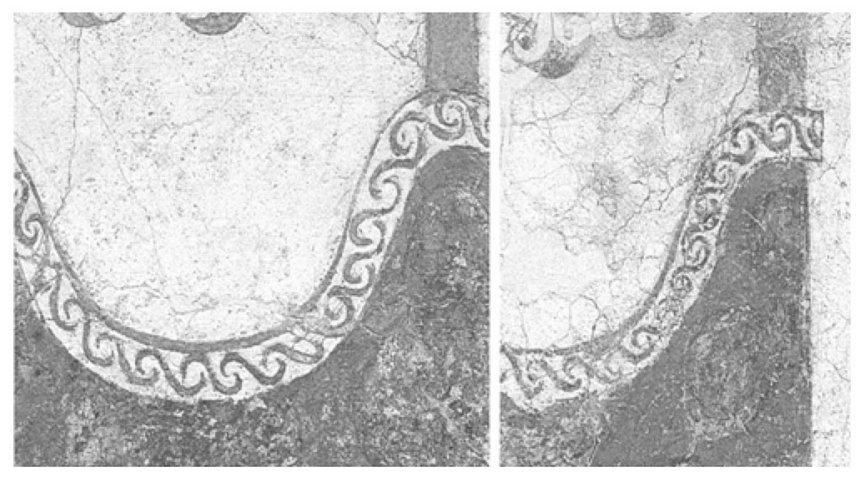

(b)

Fig. 9. The wall painting "Ikria" that almost definitely was drawn by freehand without use of stencils.

length greater than $5 \mathrm{~cm}$, then the vast majority of the corresponding object parts shown in magenta in those two figures were spotted. However, Hough's transform seems to offer inferior results to those obtained with the use of the methodology proposed in this paper. A detailed comparison of these two and other related methods will be attempted in another paper. However, we can briefly state here that the method introduced in this paper seems to manifest the following advantages:

1. It offers particularly good subpixel approximation.

2. It is faster, at least for the present application and analogous ones.

3. It is far less sensitive, if at all, to the initial conditions than the classical minimization algorithms.

4. It does not manifest essential finite precision error; we stress that the analytic least squares method greatly suffers from this type of error, at least for the application in hand.

After showing that there are no repetitions in the "Ikria" wall paintings of Fig. 9, we proceeded to apply the methodology introduced in this paper in order to test if the six types of prototype curves referred to in Section 3, i.e., the three types of spirals and the conics, appear in these wall paintings, too. Consequently, we have obtained the following results: 1) All object parts were tested against all six models, with particularly poor matching results. The corresponding approximation errors were considerably greater than the mean error encountered in the "Gathering of Crocus." 2) No single stencil of the types examined has been found to repeatedly approximate object parts of the arcs, even in the aforementioned poor sense. 


\section{CONCLUSION}

In the previous analysis, a general method was introduced that can provide strong supporting evidence that an artist or a group of artists have used a number of stencils corresponding to specific prototypes in order to draw a painting. The first step of the methodology consists of determining the so-called drawn elements, objects, and object parts. Next, a set of potential geometric stencils not a priori excluded by the civilization level of the era is spotted. A method for testing if each object part corresponds to one of these stencils is presented. Subsequently, an algorithm that provides an objective method for estimating the number of stencils used and their precise primary parameters is introduced. Application of this method to the celebrated 1650 B.C. wall painting "Gathering the crocus," unearthed in the Akrotiri excavation on the Greek island of Thera, indicates that this wall painting has been drawn via the use of seven geometric stencils. Thus, four different hyperbolae, two different ellipses, one linear (Archimedes) spiral, and seven corresponding very well constructed stencils, have been most probably used for drawing this wall painting. This method of drawing the wall paintings seems to be unique in the history of arts and of great importance for archaeology and the history of science, too. The use of precise geometric prototypes and corresponding stencils for drawing beautiful motifs and figures reveals a remarkable sense of regularity, proportionality, symmetry, and geometry.

\section{REFERENCES}

[1] C. Doumas, The Wall-Paintings of Thera. Athens: The Thera Foundation Petros Nomikos, 1999.

[2] C. Doumas, "The Elements at Akrotiri," Thera and the Aegean World III, pp. 24-30, 1990.

[3] K. Birtacha and M. Zacharioudakis, "Stereotypes in Thera Wall Paintings: Models and Patterns in the Procedure of Painting," Proc. First Int'l Symp. Wall Paintings of Thera, pp. 159-172, 2000.

[4] A. Szabo, The Beginnings of Greek Mathematics. Dordrecht: Reidel, 1968.

[5] T. Exarchakos, History of Mathematics: Mathemetics in Babylonia and Ancient Egypt, vol. A, 1997.

[6] N. Lucas, H. Bunt, P.S. Jones, and J.D. Bedient, The Historical Roots of Elementary Mathematics. Prentice-Hall, 1981.

[7] T. Heath, A History of Greek Mathematics-Volume 1: From Thales to Euclid. New York: Dover Publications, 1981.

[8] E. Spandagos, R. Spandagou, and D. Travlou, Ancient Greece Mathematicians. Athens: Aethra, 2000.

[9] T. Heath, A History of Greek Mathematics-Volume 2. New York: Dover Publications, 1981.

[10] A. Hoover, G. Jean-Baptiste, X. Jiang, P.J. Flynn, H. Bunke, D. Goldgof, K. Bowyer, D. Eggert, A. Fitzgibbon, and R. Fisher, "An Experimental Comparison of Range Image Segmentation Algorithms," IEEE Trans. Pattern Analysis and Machine Intelligence, vol. 18, no. 7, pp. 673-689, July 1996.

[11] D. Lee, S. Baek, and K. Sung, "Modified k-Means Algorithm for Vector Quantizer Design," IEEE Signal Processing Letters, vol. 4, no. 1, pp. 2-4, Jan. 1997.

[12] T. Kohonen, Self-Organization and Associative Memory. Springer Verlag, 1984.

[13] M. Werman and Z. Geyzel, "Fitting a Second Degree Curve in the Presence of Error," IEEE Trans. Pattern Analysis and Machine Intelligence, vol. 17, no. 2, pp. 207-211, Feb. 1995.

[14] D. Craig, "Fitting Curves and Surfaces with Constrained Implicit Polynomials," IEEE Trans. Pattern Analysis and Machine Intelligence, vol. 21, no. 1, pp. 31-41, Jan. 1999.

[15] K. Voss and H. Suesse, "Invariant Fitting of Planar Objects by Primitives," IEEE Trans. Pattern Analysis and Machine Intelligence, vol. 19, no. 1, pp. 80-84, Jan. 1997.
[16] S.J. Ahn, W. Rauh, H.S. Cho, and H.J. Warnecke, "Orthogonal Distance Fitting of Implicit Curves and Surfaces," IEEE Trans. Pattern Analysis and Machine Intelligence, pp. 620-638, 2002.

[17] M. Sarfraz, "Fitting Curve to Planar Digital Data," Proc. Sixth Int'l Conf. Information Visualisation (IV'02), p. 633, July 2002.

[18] P.V.C. Hough, "A Method and Means for Recognizing Complex Patterns," US Patent 3069654, 1962.

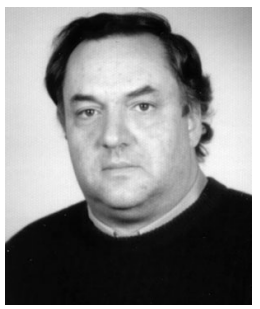

Constantin Papaodysseus received the Diploma degree in electrical and computer engineering from the National Technical University of Athens (NTUA) and the MSc degree from Manchester University, United Kingdom. He received the $\mathrm{PhD}$ degree in computer engineering from NTUA. From 1996-2000, he was an assistant professor at NTUA in the Department of Electrical and Computer Engineering. Since 2001, he has been an associate professor in the same department of NTUA. His research interests include music and speech processing and automatic recognition, image processing, applied mathematics, algorithm robustness and quantization error analysis, adaptive algorithms, biomedical engineering, etc. He has more than 35 publications in international journals and many publications in international conferences on these subjects.

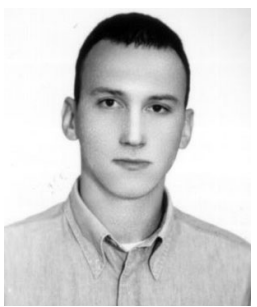

Dimitrios K. Fragoulis graduated from the Department of Electrical Engineering of the National Technical University of Athens in 1996 and he received the $\mathrm{PhD}$ degree in electrical and computer engineering in 2004. Some of his main research interests are related to the recording, transmission, and reproduction of sound. He has worked for several years on the development of audio coding and pattern matching algorithms that can find application in audio recognition problems such as audio retrieval from large databases, recognition of radio broadcasted musical recordings, automatic speaker identification, etc. Moreover, he is interested in the study of the psychological and perceptual aspects of sound, as well as the musical timbre. His current research efforts are focused on the application of image analysis in archaeology. He has published more than 12 articles in international journals.

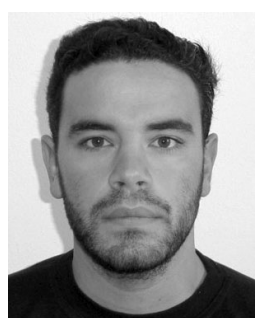

Mihalis Panagopoulos received the Diploma degree in electrical and computer engineering from the National Technical University of Athens in 2001. He has been a PhD student in the School of Electrical and Computer Engineering of the NTUA since 2003. His main research interests involve: Image processing, pattern recognition, curve fitting, finite precision error, biomedical engineering, and application of pattern recognition methods and statistics to archeology. He has four publications in international journals on these subjects.

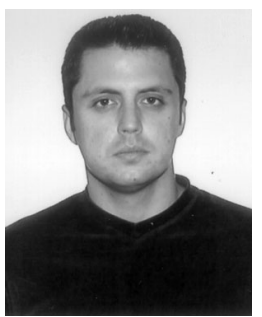

Thanasis Panagopoulos received the Diploma and MSc degree in electrical and computer engineering from the National Technical University of Athens (NTUA) in 1996. He received the $\mathrm{PhD}$ degree in computer engineering from NTUA in 2002. His research interests and recent work are on the following subjects: music and speech processing and automatic recognition, applications of information theory to archaeology, image processing, pattern recognition, and algorithms for echo cancellation, etc. He has more than 10 publications in international journals on these subjects. 


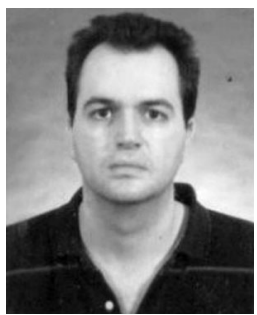

Panayiotis Rousopoulos received the Diploma degree in physics from the University of Patras in 2002. He has been a PhD student in the School of Electrical and Computer Engineering of National Technical University of Athens (NTUA) since 2002. His research interests and recent work are on the following subjects: applications of information theory to archaeology, image processing, pattern recognition, finite precision error, numerical solutions of differential equations in physics, etc. He has five publications in international journals on these subjects.

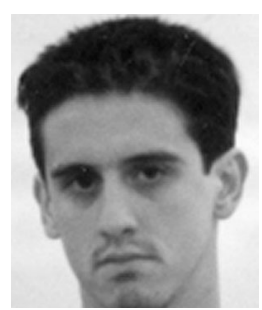

Mihalis Exarhos received the Diploma degree in electrical and computer engineering from Democritus University of Thrace in 1997. He received the $\mathrm{PhD}$ degree in computer engineering from NTUA in 2004. His research interests and recent work are in image processing, image reconstruction, and application of these in archaeology, pattern recognition, sound and music processing, etc. He has six publications in international journals and numerous publications in international conferences on these subjects.

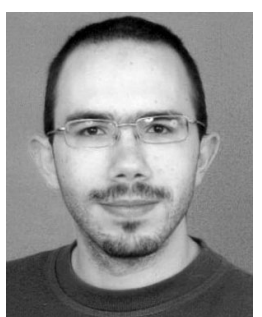

Angelos Skembris received the MEng degree in electrical and electronic engineering from Imperial College, University of London. He has been a PhD student in the School of Electrical and Computer Engineering of the National Technical University of Athens since 2001. His main research interests involve Image processing, pattern recognition, curve fitting, finite precision error, music and sound processing, biomedical engineering, and the application of pattern recognition methods and statistics to archeology. He has four publications in international journals on these subjects.

For more information on this or any other computing topic, please visit our Digital Library at www.computer.org/publications/dlib. 